\title{
Diagnostic potential of near-infrared Raman spectroscopy in the stomach: differentiating dysplasia from normal tissue
}

\author{
SK Teh', W Zheng', KY $\mathrm{Ho}^{2}, \mathrm{M} \mathrm{Teh}{ }^{3}$, KG Yeoh ${ }^{2}$ and Z Huang ${ }^{*, I}$ \\ 'Bioimaging Laboratory, Division of Bioengineering, Faculty of Engineering, National University of Singapore, Singapore 1 1 7576, Singapore; ${ }^{2}$ Department \\ of Medicine, Yoo Loo Lin School of Medicine, National University of Singapore and National University Hospital, Singapore 11 9260, Singapore; \\ ${ }^{3}$ Department of Pathology, Yoo Loo Lin School of Medicine, National University of Singapore and National University Hospital, Singapore 119074 , \\ Singapore
}

\begin{abstract}
Raman spectroscopy is a molecular vibrational spectroscopic technique that is capable of optically probing the biomolecular changes associated with diseased transformation. The purpose of this study was to explore near-infrared (NIR) Raman spectroscopy for identifying dysplasia from normal gastric mucosa tissue. A rapid-acquisition dispersive-type NIR Raman system was utilised for tissue Raman spectroscopic measurements at $785 \mathrm{~nm}$ laser excitation. A total of 76 gastric tissue samples obtained from 44 patients who underwent endoscopy investigation or gastrectomy operation were used in this study. The histopathological examinations showed that 55 tissue specimens were normal and 21 were dysplasia. Both the empirical approach and multivariate statistical techniques, including principal components analysis (PCA), and linear discriminant analysis (LDA), together with the leave-one-sample-out crossvalidation method, were employed to develop effective diagnostic algorithms for classification of Raman spectra between normal and dysplastic gastric tissues. High-quality Raman spectra in the range of $800-1800 \mathrm{~cm}^{-1}$ can be acquired from gastric tissue within $5 \mathrm{~s}$. There are specific spectral differences in Raman spectra between normal and dysplasia tissue, particularly in the spectral ranges of $1200-1500 \mathrm{~cm}^{-1}$ and $1600-1800 \mathrm{~cm}^{-1}$, which contained signals related to amide III and amide I of proteins, $\mathrm{CH}_{3} \mathrm{CH}_{2} \mathrm{twisting}$ of proteins/nucleic acids, and the $\mathrm{C}=\mathrm{C}$ stretching mode of phospholipids, respectively. The empirical diagnostic algorithm based on the ratio of the Raman peak intensity at $875 \mathrm{~cm}^{-1}$ to the peak intensity at $1450 \mathrm{~cm}^{-1}$ gave the diagnostic sensitivity of $85.7 \%$ and specificity of $80.0 \%$, whereas the diagnostic algorithms based on PCA-LDA yielded the diagnostic sensitivity of $95.2 \%$ and specificity 90.9\% for separating dysplasia from normal gastric tissue. Receiver operating characteristic (ROC) curves further confirmed that the most effective diagnostic algorithm can be derived from the PCA-LDA technique. Therefore, NIR Raman spectroscopy in conjunction with multivariate statistical technique has potential for rapid diagnosis of dysplasia in the stomach based on the optical evaluation of spectral features of biomolecules.
\end{abstract}

British Journal of Cancer (2008) 98, 457-465. doi: I0.1038/sj.bjc.6604I76 www.bjcancer.com

Published online 15 January 2008

(c) 2008 Cancer Research UK

Keywords: dysplasia; near-infrared Raman spectroscopy; optical diagnosis; stomach; principal components analysis; linear discriminant analysis

Gastric cancer is currently the fourth most common malignancy, and also the second leading cause of cancer deaths in humans worldwide (Axon, 2006; Clark et al, 2006). In Singapore, despite a falling incidence rate, gastric cancer still remains the fourth most common cancer (Teh et al, 2002). Many of these patients will die mainly because of nodal and metastatic disease present at the time of initial diagnosis. Early detection and localisation with immediate removal and treatment of premalignant lesions (e.g., dysplasia) (Clark et al, 2006) is crucial to improving patients' survival. However, early identification of dysplasia in the stomach can be very difficult to detect by conventional diagnostic methods such as white-light endoscope, as the white-light endoscopy

*Correspondence: Dr Z Huang, Bioimaging Laboratory, Division of Bioengineering, Faculty of Engineering, National University of Singapore, 9 Engineering Drive I, Singapore 1 17576, Singapore;

E-mail: biehzw@nus.edu.sg

Received 7 September 2007; revised 4 December 2007; accepted 4 December 2007; published online 15 January 2008 heavily relies on the visual observation of gross morphological changes of pathologic tissues, leading to a poor diagnostic accuracy.

In the past decade, optical spectroscopic methods such as Raman spectroscopy, which makes use of inelastic light scattering process to capture 'fingerprints' of specific molecular structures and conformations of a given tissue or disease state, have been comprehensively investigated for cancer and precancer diagnosis and evaluation in humans (Frank et al, 1995; Mahadevan-Jansen and Richards-Kortum, 1996; Gniadecka et al, 1997; Mahadevan-Jansen et al, 1998a; Bakker Schut et al, 2000; Shim et al, 2000; Stone et al, 2000; Caspers et al, 2003; Huang et al, 2003). Near-infrared (NIR) Raman spectroscopy has certain advantages over Fourier transform IR spectroscopy in tissue diagnosis, such as relative insensitivity to water, and a deeper penetration in the tissue using NIR excitation light. As such, NIR Raman spectroscopy has received great interest for in vitro and in vivo diagnosis of malignancies in a variety of organs (Mizuno et al, 1994; Frank et al, 1995; Gniadecka et al, 1997; Mahadevan-Jansen et al, 1998a; Shim et al, 2000; Stone et al, 2000; 
Caspers et al, 2003; Huang et al, 2003). These investigations show that specific spectral features of Raman spectra could be used to correlate with the molecular and structural changes of tissue associated with neoplastic transformation (Mahadevan-Jansen and Richards-Kortum, 1996; Gniadecka et al, 1997; Mahadevan-Jansen et al, 1998a; Stone et al, 2000; Huang et al, 2003). For instance, using NIR Raman technique, the diagnostic sensitivity and specificity of 82 and $92 \%$, respectively, can be achieved for differentiation between precancerous and benign cervical tissues in vitro (Mahadevan-Jansen et al, 1998a). Near-infrared Raman spectroscopy has also been applied for in vivo precancer and cancer diagnosis and detection of organs such as cervix, skin, colon, and oesophagus (Mahadevan-Jansen et al, 1998b; Shim et al, 2000; Huang et al, 2001; Utzinger et al, 2001). The Raman spectroscopic characterisation and discrimination of malignancy in the stomach have also been investigated (Ling et al, 2002; Stone et al, 2002; Kumar et al, 2007). However, to date, application of Raman spectroscopy on early diagnosis of gastric precancer (dysplasia) has not yet been reported in detail in literature.

Despite the great advantages that NIR Raman spectroscopy could offer, there are technical challenges to overcome. For instance, achieving a high signal-to-noise $(\mathrm{S} / \mathrm{N})$ ratio, while avoiding interference from silica Raman signals in a rapid manner can be difficult for in vivo tissue Raman measurements (Bakker Schut et al, 2000; Huang et al, 2001; Utzinger et al, 2001). This is because tissue Raman scattering is inherently very weak, and the fibre-optic probes used to collect in vivo signals exhibit strong silica Raman scattering in the fingerprint region. Also, the integration times and irradiance powers for in vivo Raman measurements must be limited for practical and safety reasons. Furthermore, Raman spectral differences are usually subtle with apparent spectral overlappings and variations in intensity between different tissue types, and thus developing effective diagnosis algorithms are highly required for effective tissue classification (Bakker Schut et al, 2000; Shim et al, 2000; Huang et al, 2003, 2004; Molckovsky et al, 2003; Lau et al, 2005). The primary aims of this study were to characterise Raman properties of gastric tissues and to assess the feasibility of using a rapid fibre-optic NIR Raman spectroscopy for precancer diagnosis of gastric tissue. Both the empirical approach and the multivariate statistical techniques, including principal components analysis (PCA) and linear discriminant analysis (LDA), were employed to develop effective diagnostic algorithms for differentiations between normal and dysplasia tissue in the stomach.

\section{MATERIALS AND METHODS}

\section{Raman instrumentation}

The instrument used for tissue Raman spectroscopic studies has been described in detail elsewhere (Huang et al, 2001). Briefly, this system consists of a 785-nm diode laser, a transmissive imaging spectrograph with a Kaiser holographic grating, an NIR-optimised back-illuminated, deep-depletion charge-coupled device (CCD) detector (Princeton Instruments, Trenton, NJ, USA), and an in-house developed fibre optic Raman probe. The 785-nm laser is coupled to a $100-\mu \mathrm{m}$ core diameter fibre $(\mathrm{NA}=0.22)$ and the fibre is connected to the Raman probe via an SMA connector. The Raman probe was designed to maximise the collection of tissue Raman signals while reducing the interference of Rayleigh scattered light, fibre fluorescence, and silica Raman signals. One optical arm of the probe consists of a collimating lens, a bandpass filter (785 $\pm 2.5 \mathrm{~nm}$, Chroma Technology Corp., Rockingham, VT, USA), and a focusing lens to deliver the laser light onto the tissue. The other arm of the probe equipped with collimating and refocusing lenses and a holographic notch plus filter (optical density $>6.0$ at $785 \mathrm{~nm}$; Kaiser) is used for collecting tissue Raman
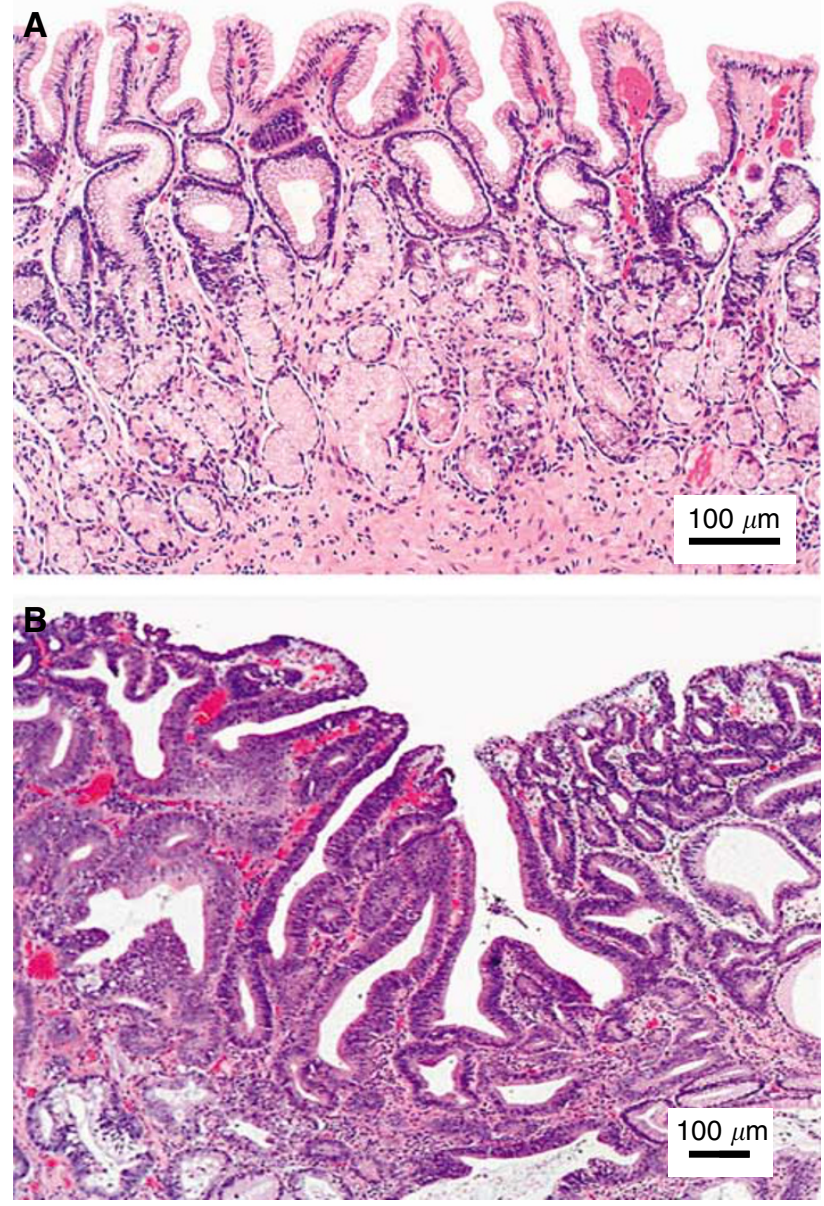

Figure I Photomicrographs of the haematoxylin and eosin (H\&E)stained tissue sections of gastric tissues (A) normal and (B) dysplasia (high-grade dysplasia of the antrum). Scale bar: $100 \mu \mathrm{m}$.

signals. The holographic notch filter was placed between the two lenses to block the Rayleigh scattered excitation laser light while passing the frequency-shifted tissue Raman signal. The refocusing lens then focused the filtered beam onto the circular end of the fibre bundle $(58 \times 100 \mu \mathrm{m}$ core diameter fibres, NA $=0.22)$. Tissue Raman photons collected by the fibre bundle in the Raman probe are fed into the entrance of the transmissive spectrograph along a parabolic curve, and the holographic grating disperses the incoming light onto the liquid nitrogen-cooled CCD array detector controlled by a principal component (PC) (Huang et al, 2001, 2003). The tissue Raman spectra associated with autofluorescence background are displayed on the computer screen in real time and can be saved for further analysis. The system acquired Raman spectra over the wavenumber range of $800-1800 \mathrm{~cm}^{-1}$, and each spectrum was acquired within $5 \mathrm{~s}$ with light irradiance of $1.56 \mathrm{~W} \mathrm{~cm}{ }^{-2}$. The spectral resolution of the system is $4 \mathrm{~cm}^{-1}$. All wavelength-calibrated spectra were also corrected for the wavelength dependence of the system using a standard lamp (RS-10; EG\&G Gamma Scientific, San Diego, CA, USA).

\section{Gastric tissue samples}

A total of 76 gastric tissue samples were collected from 44 patients (21 men and 23 women with a median age of 62 years) who underwent gastrectomy or endoscopic biopsies with clinically suspicious lesions. All patients preoperatively signed an informed consent, permitting the investigative use of the tissues, and this study was approved by the Ethics Committee of the National 
Healthcare Group (NHG) of Singapore. After biopsies or surgical resections, tissue samples were immediately sent to the laboratory for Raman measurements. After spectral measurements, the tissue samples were fixed in $10 \%$ formalin solution and then submitted back to the hospital for histopathologic examination. The histopathogical examinations were conducted by a specialist gastrointestinal pathologist, and the results showed that among the 76 homogenous gastric tissue samples with clearly defined pathologies, 55 tissue specimens were normal, and 21 were dysplasia (8 low-grade and 13 high-grade dysplasia). Figure 1 shows the comparison of haematoxylin and eosin (H\&E)-stained tissue sections of normal and dysplastic gastric tissues, illustrating the crowding of irregularly shaped glands with branching and prominent nuclear abnormalities (including irregular and thickened nuclear membranes and irregular chromatin) in dysplasia mucosa. Note that the gastric tissue samples were approximately $3 \times 3 \times 2 \mathrm{~mm}$ in size, and the $785-\mathrm{nm}$ laser light with a beam size of $1 \mathrm{~mm}$ was focused on the tissue surface to mimic the in vivo clinical measurements. The tissue surface measured was then marked and stained for tissue pathology. After comparing with pathologic results, only those Raman spectra that were correctly acquired from the surfaces of gastric tissues were used for data analysis. To reduce the spectral measurement errors in this study, the average spectrum of five repeated Raman measurements on the same tissue site of each tissue sample was used for tissue classification.

We have measured the thickness of various layers of typical normal and dysplastic tissue sections and constructed a gastric tissue model, which consisted of mucosa, submucosa, muscularis propria, and serosa layers with a total thickness of $1.5 \mathrm{~mm}$ for normal tissue, while the dysplasia tissue consisted of thickening mucosa, submucosa, muscle propria, and serosa layers with a total thickness of $2 \mathrm{~mm}$ (Sabet et al, 2003). Optical parameters (absorption coefficient, scattering coefficient, scattering anisotropy, and refractive index) from literature (Bashkatov et al, 2007) were composed for these layers to set up a tissue optics model for Monte Carlo simulation of light penetration into these model tissues. The simulation results (data not shown) showed that the 785-nm light penetrated down to about $750 \mu \mathrm{m}$ beneath the surface of normal tissue, which was within the muscularis propria layer. For dysplasia tissue, the $785-\mathrm{nm}$ light penetrated down to about $780 \mu \mathrm{m}$ beneath the surface, which was also well within the muscularis propria. It is expected that most of the spectral signals from normal tissue came from mucosa, submucosa layers with some small contributions from the muscularis propria, and serosa layer, because tissue layers closing to the surface encountered stronger excitation light and the generated Raman scattered light was also easier to escape out of the tissue. Similarly, most of the spectral signals from dysplasia came from mucosa, submucosal layers. The exact percentile contributions of various tissue layers can be obtained by more detailed modelling of not only the excitation light propagation but also the Raman scattered light propagation in tissue.

\section{Data preprocessing}

The raw spectra acquired from gastric tissue in the $800-1800 \mathrm{~cm}^{-1}$ range represented a combination of prominent tissue autofluorescence, weak tissue Raman scattering signals, and noise. Thus, the raw spectra were preprocessed by adjacent five-point smoothing to reduce noise. A fifth-order polynomial (Huang et al, 2003) was found to be optimal for fitting the broad autofluorescence background in the noise-smoothed spectrum, and this polynomial was then subtracted from the raw spectrum to yield the tissue Raman spectrum alone. Each of background-subtracted Raman spectrum was also normalised to the integrated area under the curve from 800 to $1800 \mathrm{~cm}^{-1}$ to enable a better comparison of the spectral shapes and relative peak intensities among the different tissue samples.

\section{Empirical approach}

Nonparametric diagnostic algorithms based on peak intensities, spectral bandwidths, and/or peak ratios have been widely employed in literature to correlate the variations of tissue spectra with tissue pathology in a simple and straightforward fashion (Mahadevan-Jansen and Richards-Kortum, 1996; MahadevanJansen et al, 1998a; Huang et al, 2003). In this study, the empirical diagnostic algorithm based on the ratio of the Raman peak intensity at $875 \mathrm{~cm}^{-1}$ for hydroxyproline to the peak intensity at $1450 \mathrm{~cm}^{-1}$ for $\mathrm{CH}_{2}$ proteins/lipids (Stone et al, 2000; Huang et al, 2003) was selected for tissue classification. The unpaired Student's $t$-test was used to test the difference of Raman intensity ratio $\left(\mathrm{I}_{875} / \mathrm{I}_{1450}\right)$ between normal and dysplasia tissues. For the assessment of diagnostic sensitivity and specificity, histopathological results were regarded as the gold standard.

\section{Multivariate analysis}

The high dimension of Raman spectral space (each Raman spectrum ranging from 800 to $1800 \mathrm{~cm}^{-1}$ with a set of 544 intensities) will result in computational complexity and inefficiency in optimisation and implementation of the LDA algorithms. As such, PCA was first performed on tissue Raman data set to reduce the dimension of Raman spectral space while retaining the most diagnostically significant information for tissue classification. To eliminate the influence of inter- and/or intra-subject spectral variability on PCA, the entire spectra were standardised so that the mean of the spectra was zero, and the standard deviation (s.d.) of all the spectral intensities was one. Mean centring ensures that the (PCs form an orthogonal basis (Lachenbruch and Mickey, 1968; Devore, 1992). The standardised Raman data sets were assembled into data matrices with wavenumber columns and individual case rows. Thus, PCA was performed on the standardised spectral data matrices to generate PCs comprising a reduced number of orthogonal variables that accounted for most of the total variance in original spectra. Each loading vector is related to the original spectrum by a variable called the PC score, which represents the weight of that particular component against the basis spectrum. Principal component scores reflect the differences between different classes. Unpaired Student's $t$-test (Devore, 1992) was used to identify the most diagnostically significant PCs $(P<0.05)$. These significant PC scores are lastly selected as input for the development of LDA algorithms for binary-class classification. Linear discriminant analysis determines the discriminant function that maximises the variances in the data between groups while minimising the variances between members of the same group. The performance of the diagnostic algorithms rendered by the LDA models for correctly predicting the tissue groups (i.e., normal $v s$ dysplasia) was estimated in an unbiased manner using the leave-one-sampleout, cross-validation method (Lachenbruch and Mickey, 1968; Dillion and Goldstein, 1984) on all model spectra. In this method, one sample (i.e., one spectrum) was held out from the data set, and the entire algorithm including PCA and LDA was redeveloped using the remaining tissue spectra. The algorithm was then used to classify the withheld spectrum. This process was repeated until all withheld spectra were classified.

To compare the performance of the empirical and multivariate approaches for tissue classification using the same Raman data set, receiver operating characteristic (ROC) curves were generated by successively changing the thresholds to determine correct and incorrect classifications for all tissue samples.

\section{RESULTS}

To assess intrasample variability, multiple Raman measurements $(n=5)$ on each of normal and dysplasia gastric tissues were 
made at different locations of the same samples. Figure 2 shows an example of the mean normalised Raman spectra \pm 1 s.d. measured from a normal (A) and a dysplasia (B) gastric tissue, respectively. The overall spectral intensities varied by $30 \%$ about the mean for normal tissue, and by $20 \%$ for dysplasia tissue. However, the relative Raman peak heights, shapes, and positions showed little intrasample variability for either normal or dysplasia tissue, indicating the relative homogeneity of tissue samples used in this study.

The intersample variations of all Raman spectra from normal or dysplasia tissues obtained from 44 patients were also studied. Figure 3 shows the mean normalised Raman spectra \pm 1 s.d. from normal $(n=55)$ and dysplasia $(n=21)$ gastric tissues. The overall spectral intensities varied by $20-30 \%$ about the mean for normal tissue, and by $30-60 \%$ for dysplasia tissue. Although there are only some small changes in spectral shapes and Raman peak positions between normal and dysplasia tissue, there is a large overlap in intensity throughout the entire spectral range of Raman spectra between the two tissue types. This indicates a relatively large variability of tissue constituents among different subjects. Hence, there is a need to develop effective diagnostic algorithms for accurate classification of Raman spectra between normal and dysplastic tissues.

Figure 4A shows the comparison of mean normalised Raman spectra between normal and dysplasia gastric tissue. The prominent Raman peaks located at around 875, 1004, 1100, 1210, $1265,1335,1450,1655$, and $1745 \mathrm{~cm}^{-1}$ are observed in gastric tissue, which can be attributed to the biochemical bonds of hydroxyproline, the phenylalanine (an essential amino acid) ring breathing mode, phospholipids (stretching C-C skeletal vibrations in the gauche conformation), tryptophan (an essential amino acid) and phenylalanine stretching $\left(\mathrm{C}^{-} \mathrm{C}_{6} \mathrm{H}_{5}\right)$ mode, amide III ( $\mathrm{C}-\mathrm{N}$ stretching mode of proteins, indicating mainly $\alpha$-helix conformation), $\mathrm{CH}_{3} \mathrm{CH}_{2}$ twisting mode of proteins and nucleic acids, $\mathrm{CH}_{2}$ bending mode of proteins and lipids, the protein amide $\mathrm{I}$ band $(\mathrm{C}=\mathrm{O}$ stretching mode of proteins, indicating mainly $\alpha$-helix conformation), and the $\mathrm{C}=\mathrm{O}$
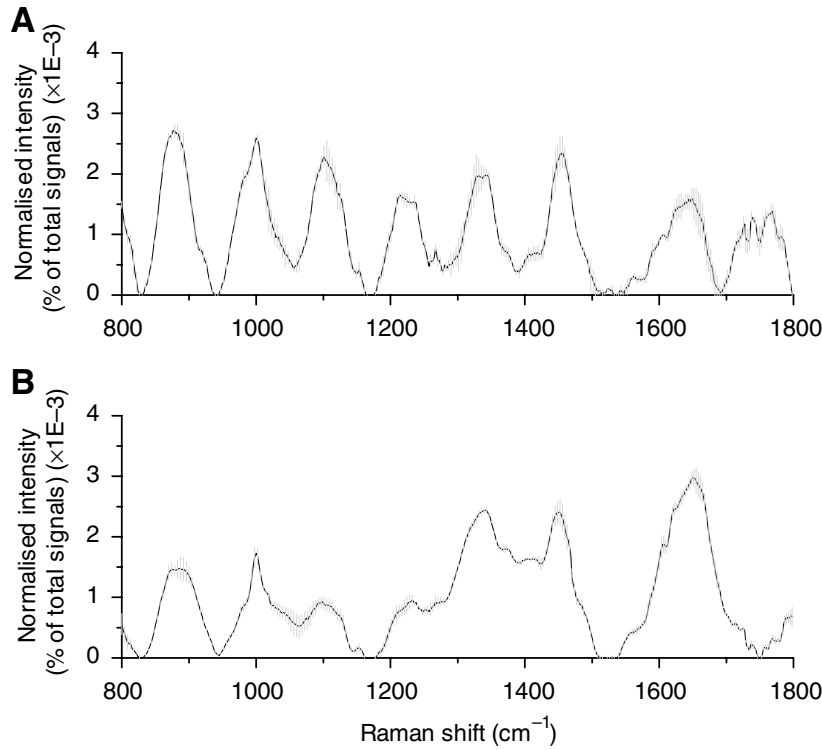

Figure 2 Mean normalised gastric Raman spectra (solid line) \pm I s.d. (grey area) obtained from a normal tissue $(\mathbf{A})$ and a dysplasia tissue $(\mathbf{B})$ by multiple measurements $(n=5)$ at various locations for each sample. Each spectrum was normalised to the integrated area under the curve to correct for variations in absolute spectral intensity. All spectra were acquired in $5 \mathrm{~s}$ with 785-nm excitation and corrected for spectral response of the system. stretching mode of phospholipids, respectively (Dollish et al, 1974; Mizuno et al, 1994; Frank et al, 1995; Mahadevan-Jansen and Richards-Kortum, 1996; Gniadecka et al, 1997; Mahadevan-Jansen et al, 1998a, b; Bakker Schut et al, 2000; Shim et al, 2000; Stone et al, 2000, 2002; Huang et al, 2001, 2003; Utzinger et al, 2001; Caspers et al, 2003). The intensity differences between the two tissue types are remarkable (Figure 4B). For instance, dysplasia tissues show lower intensities at 875, 1004, 1100, 1210, and
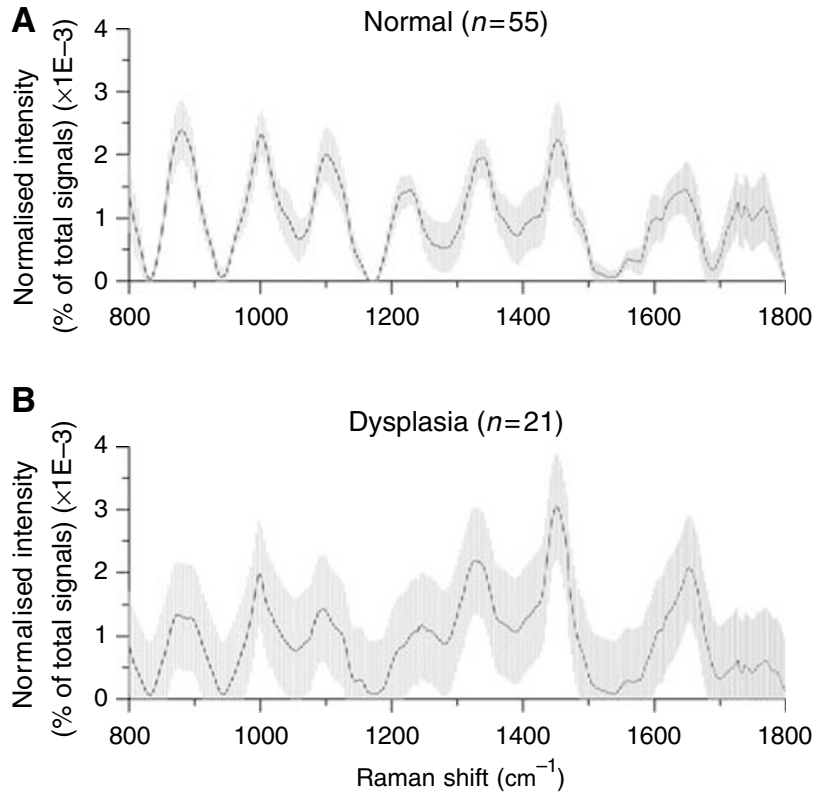

Figure 3 Mean normalised gastric Raman spectra \pm I s.d. (shaded area) from (A) normal tissues $(n=55)$ and (B) dysplasia tissues $(n=21)$ illustrating the intensity variations in major Raman peaks of $20-30 \%$ for normal tissues whereas of 30-60\% for dysplasia tissues.
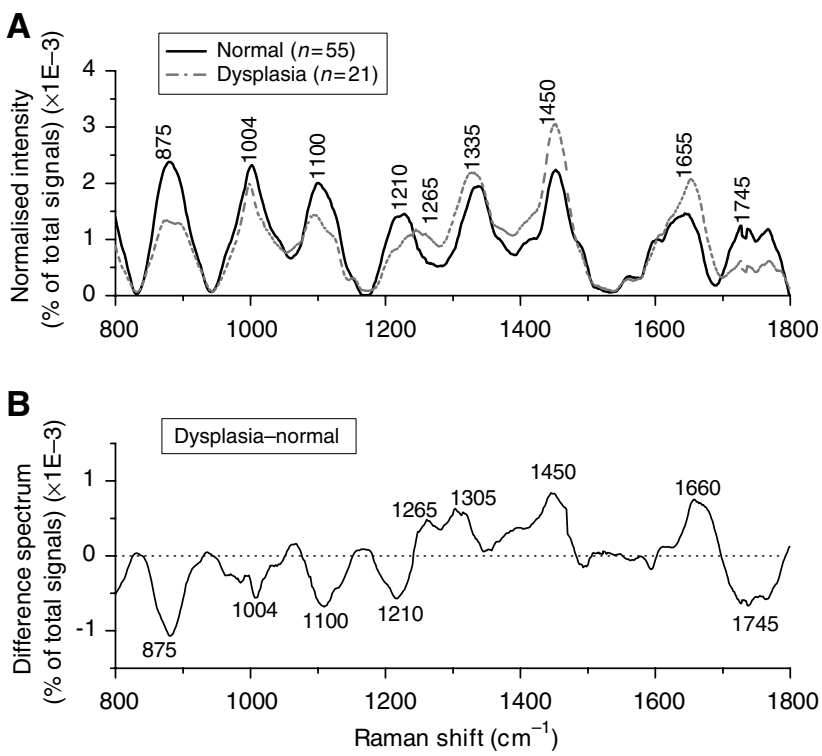

Figure 4 (A) Comparison of the mean normalised Raman spectra of normal $(n=55)$ and dysplasia $(n=21)$ tissues. (B) Difference spectrum calculated from the mean Raman spectra of normal and dysplasia tissue (i.e., the mean normalised Raman spectrum of dysplasia tissue minus the mean normalised Raman spectrum of normal tissue). 
$1745 \mathrm{~cm}^{-1}$, while higher at $1265,1305,1450$, and $1660 \mathrm{~cm}^{-1}$, compared with normal tissue. This indicates that there is an increase or decrease in the percentage of a certain type of biomolecules relative to the total Raman-active constituents in dysplasia tissue. There are also obvious changes of Raman peak shifts and bandwidths in the spectral ranges of $1200-1500 \mathrm{~cm}^{-1}$ and $1600-1800 \mathrm{~cm}^{-1}$, which are related to the amide III and amide I of proteins, $\mathrm{CH}_{3} \mathrm{CH}_{2}$ twisting of proteins/nucleic acids, and $\mathrm{C}=\mathrm{C}$ stretching of phospholipids for dysplasia. These spectral differences between normal and dysplasia tissues can be viewed more

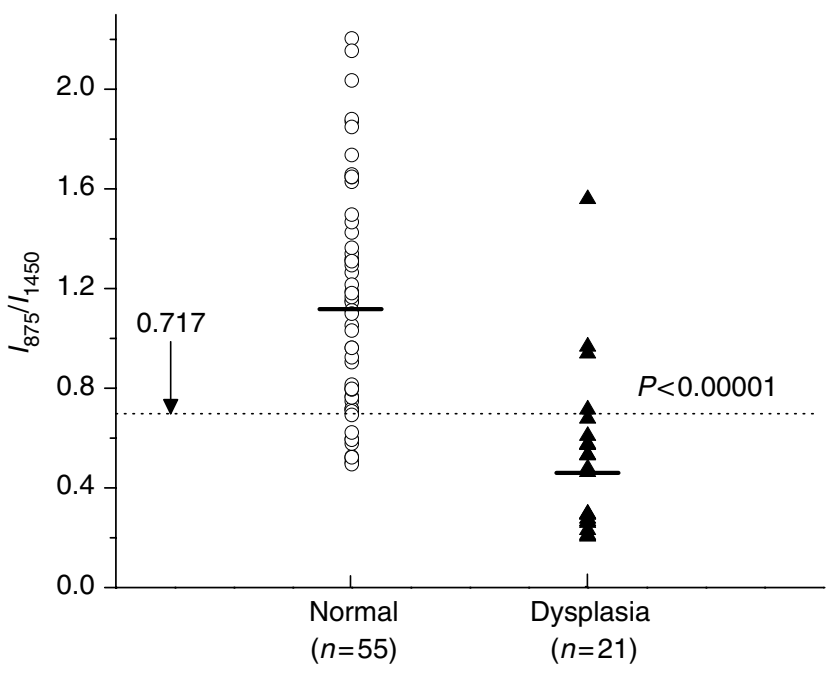

Figure 5 Scatter plot of the intensity ratio of Raman signals at 875 and $1450 \mathrm{~cm}^{-1}$, as measured for each sample and classified according to the histological results. The decision line $\left(I_{875} / I_{1450}=0.717\right)$ separates dysplasia tissue from normal tissue with a sensitivity of $85.7 \%$ (I8/2I) and specificity of $80.0 \%(44 / 55)$ clearly using the difference spectrum as shown in Figure $4 \mathrm{~B}$. The difference spectrum reveals the changes of prominent Raman peaks occurring in dysplasia gastric tissue, confirming a potential role of Raman spectroscopy for precancer diagnosis in the stomach.

The empirical analysis based on the intensity ratio of prominent Raman bands is employed for tissue diagnosis. Figure 5 shows the scatter plot of the ratio of Raman intensity at $875 \mathrm{~cm}^{-1}$ to that at $1450 \mathrm{~cm}^{-1}$ grouped according to tissue pathologic types. The mean value (mean \pm s.d.) of this ratio for normal tissues $(1.13 \pm 0.46$, $n=55)$ is significantly different from the mean value for dysplastic tissues $(0.52 \pm 0.33, n=21)$ (unpaired two-sided Student's $t$-test, $P<0.00001)$. The decision line $\left(\mathrm{I}_{875} / \mathrm{I}_{1450}=0.717\right)$ discriminates dysplasia tissue from normal gastric tissue with a sensitivity of $85.7 \%$ and a specificity of $80.0 \%$.

We also employ the multivariate statistical method (e.g., PCA and LDA) by incorporating the entire Raman spectrum to determine the most diagnostically significant Raman features for improving tissue analysis and classification. Unpaired two-sided Student's $t$-test on the obtained PC scores showed that there were four PCs (PC1, PC2, PC4, and PC5) that were diagnostically significant $(P<0.05)$ for discriminating dysplasia tissue from normal tissue. Figure 6 displays the four significant PC scores calculated from PCA on the Raman spectra. The first PC accounts for the largest variance (e.g., $42.6 \%$ of the total variance), whereas the successive PCs describe the spectral features that contribute progressively smaller variances. Some PC features (Figures 6A-D), such as peaks, troughs, and spectral shapes are similar to those of tissue Raman spectra in Figure 4.

Figure 7 shows the correlations between the diagnostically significant PC scores for normal and dysplastic gastric tissue, illustrating the utility of PC scores for classification of Raman spectra between different tissue types. Normal and dysplasia tissues can be largely clustered into two separate groups based on different combinations of significant PCs, and the corresponding separation lines (i.e., diagnostic algorithms) in Figures $7 \mathrm{~A}-\mathrm{F}$ classify dysplasia from normal tissue with

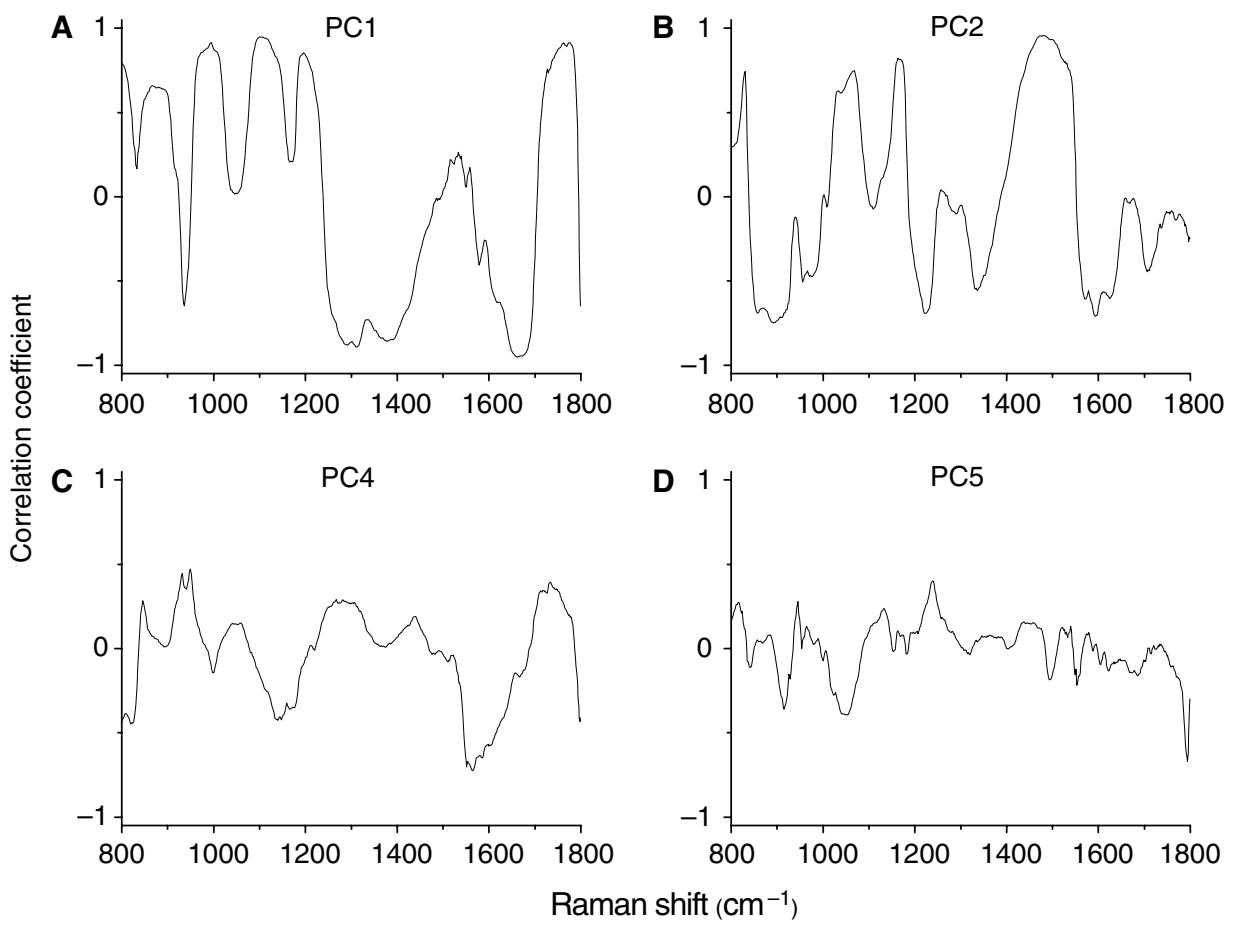

Figure 6 The first four diagnostically significant principal components (PCs) accounting for about 78.5\% of the total variance calculated from Raman spectra (PCI $-42.6 \%, P C 2-25.4 \%$, PC4 - 7.9\%, and PC5 - 2.6\%), revealing the diagnostically significant spectral features for tissue classification. 

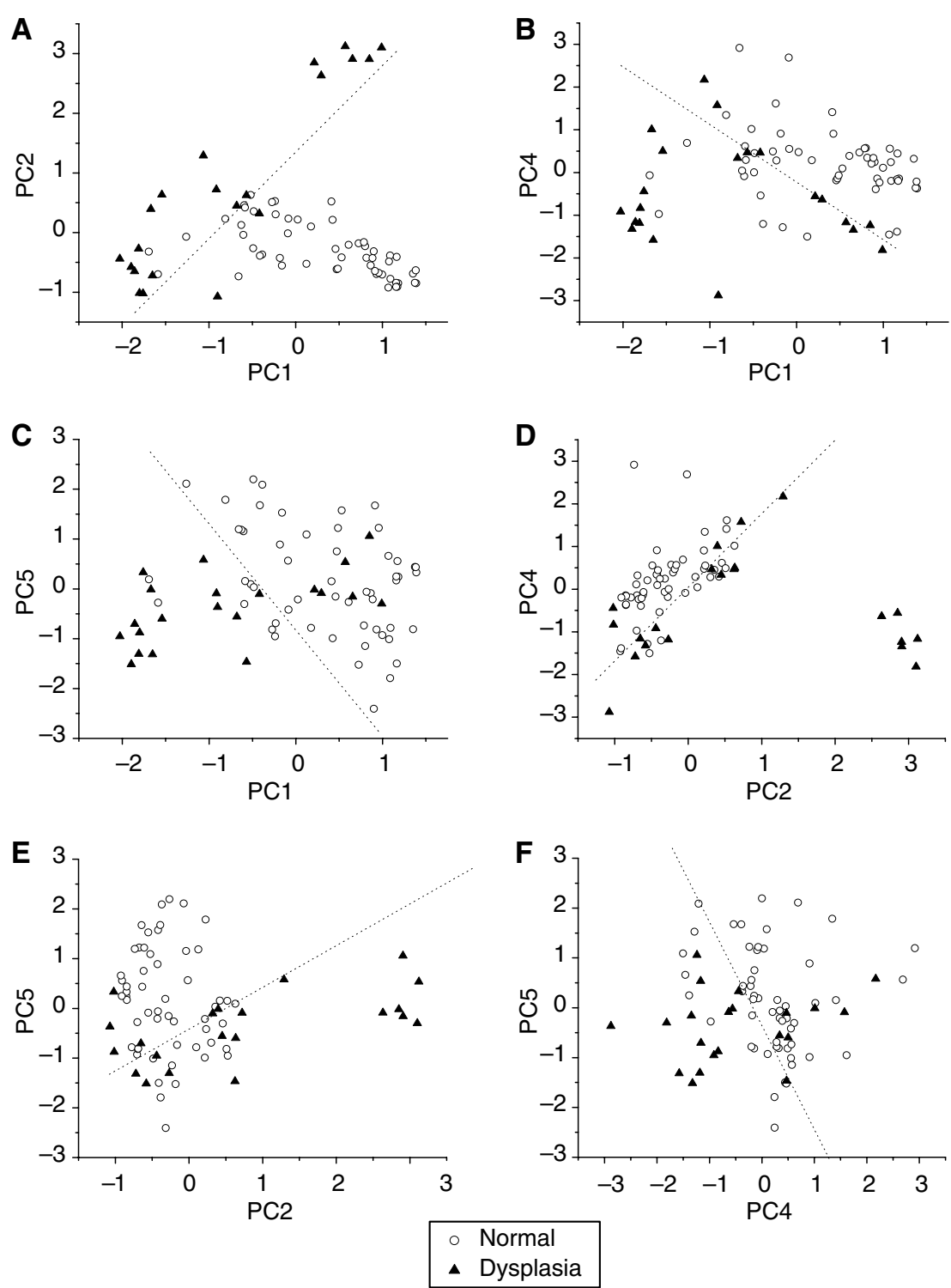

Figure 7 Scatter plots of the diagnostically significantly principal component (PC) scores for normal and dysplastic gastric tissue derived from Raman spectra, (A) PCI vs PC2; (B) PCI vs PC4; (C) PCI vs PC5; (D) PC2 vs PC4; (E) PC2 vs PC5; and (F) PC4 vs PC5. The dotted lines (PC2 = I.46 $\mathrm{PCI}+1.34 ; \mathrm{PC} 4=-1.32 \mathrm{PCl}+0.94 ; \mathrm{PC} 5=-2.16 \mathrm{PCl}-0.89 ; \mathrm{PC} 4=1.74 \mathrm{PC} 2+0.12 ; \mathrm{PC} 5=0.84 \mathrm{PC} 2-0.381 ;$ and $\mathrm{PC} 5=-2.05 \mathrm{PC} 4-0.29)$ as diagnostic algorithms classify dysplasia from normal with sensitivity of $90.5 \%$ (I9/2I), 76.2\% (I6/2I), 7I.4\% (I5/2I), 8I.0\% (I7/2I), 7I.4\% (I5/2I), and 7I.4\% (I5/2I); specificity of $90.9 \%$ (50/55), 80.0\% (44/55), 83.6\% (46/55), 80.0\% (44/55), 72.7\% (40/55), and 72.7\% (40/55), respectively. Circle (O): normal; Triangle $(\mathbf{\Lambda})$ : dysplasia.

the sensitivity of $90.5 \%, 76.2 \%, 71.4 \%, 81.0 \%, 71.4 \%$, and $71.4 \%$; specificity of $90.9 \%, 80.0 \%, 83.6 \%, 80.0 \%, 72.7 \%$, and $72.7 \%$, respectively. These results show that selection of different combinations of significant PCs will give different levels of accuracy for tissue classification.

To further improve tissue diagnosis, all the four diagnostically significant PCs were loaded into the LDA model for generating effective diagnostic algorithms for tissue classification. Figure 8 shows the classification results based on PCA-LDA technique together with leave-one-spectrum-out, crossvalidation method. The PCA-LDA diagnostic algorithms yielded the diagnostic sensitivity of $95.2 \%$ and specificity $90.9 \%$ for separating dysplasia from normal gastric tissues.

To evaluate and compare the performance of the PCA-LDAbased diagnostic algorithms derived from all the significant PCs of tissue Raman data set against the empirical approach-based diagnostic algorithm derived from the intensity ratio of $\mathrm{I}_{875} / \mathrm{I}_{1450}$, the ROC curves (Figure 9) were generated from the scatter plots in Figures 5 and 8 at different threshold levels, displaying the discrimination results using both diagnostic algorithms. A comparative evaluation of the ROC curves indicates that PCA-LDA-based diagnostic algorithm gives more effective diagnostic capability for detection of gastric dysplasia from normal gastric tissues, as illustrated by the improvement in the diagnostic sensitivity and specificity. The integration areas under the ROC curves are 0.98 and 0.88 , respectively, for PCA-LDA-based diagnostic algorithms and the nonparametric intensity ratio algorithm, respectively. These results demonstrate that PCA-LDA-based diagnostic algorithms that utilised the entire spectral features of Raman spectra yield a better diagnostics accuracy than the empirical approach. 


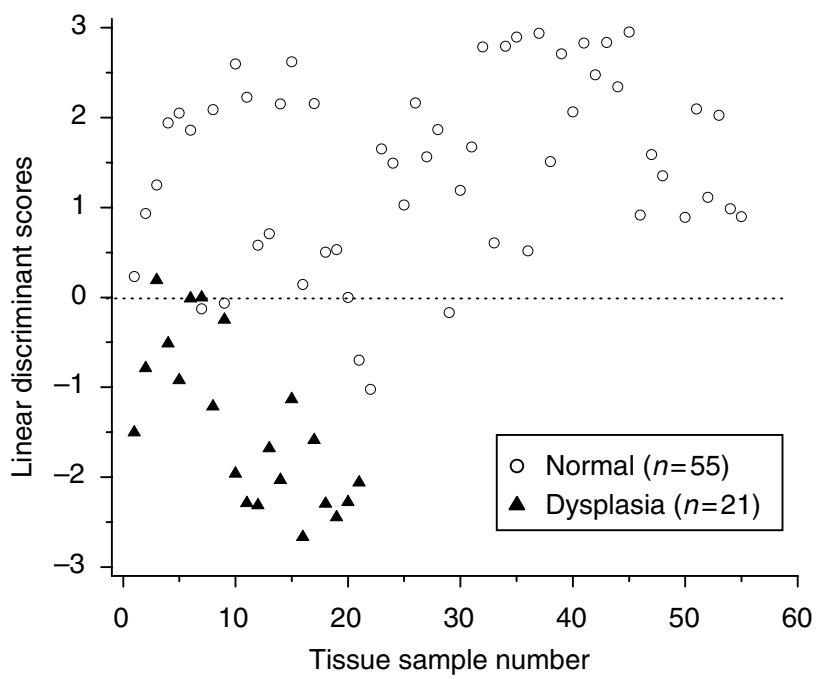

Figure 8 Scatter plot of the linear discriminant scores for the normal and dysplasia categories using the PCA-LDA technique together with leaveone-spectrum-out, cross-validation method. The separate line yields a diagnostic sensitivity of $95.2 \%(20 / 21)$ and specificity of $90.9 \%$ (50/55) for differentiation between normal and dysplasia tissue. LDA, linear discriminant analysis; PCA, principal components analysis.

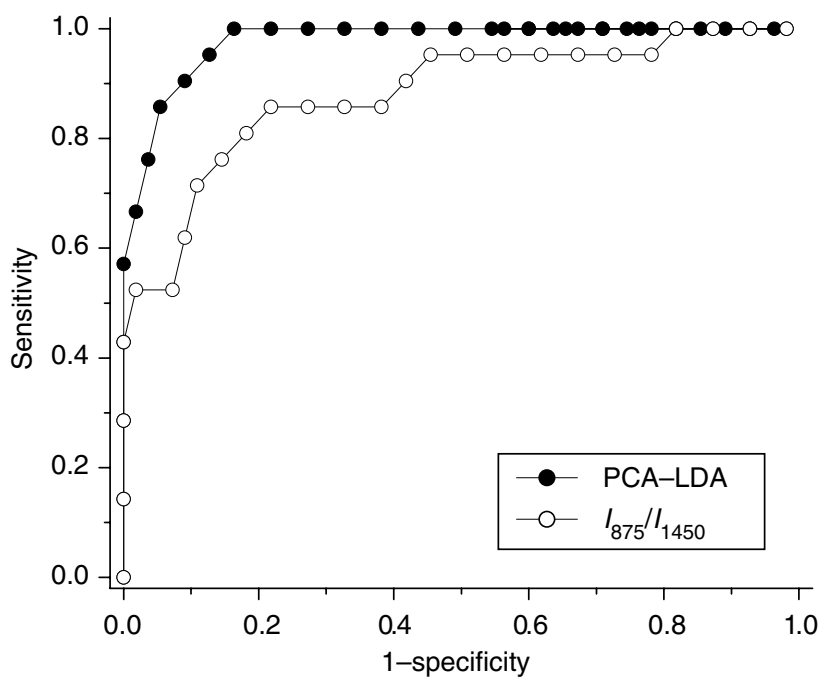

Figure 9 Comparison of receiver operating characteristic (ROC) curves of discrimination results for Raman spectra utilising the PCA-LDA-based spectral classification with leave-one-spectrum-out, cross-validation method and the empirical approach using Raman intensity ratio of $I_{875} / I_{1450}$. The integration areas under the ROC curves are 0.98 and 0.88 for PCA-LDAbased diagnostic algorithm and intensity ratio algorithm, respectively, demonstrating the efficacy of PCA-LDA algorithms for tissue classification. LDA, linear discriminant analysis; PCA, principal components analysis.

\section{DISCUSSION}

The current gold standard for clinical diagnosis of gastric dysplasia is through histological observation by the pathologist, on the extent of cytological and architectural abnormalities of the histologically prepared tissue samples (Lauwers and Riddell, 1999). These abnormalities involve much molecular alterations, which could also be tapped upon for diagnosis, most importantly during routine endoscopic inspection (Lauwers and Riddell, 1999). Hence, Raman spectroscopy, which is capable of providing rich biochemical and biomolecular information about tissue, may be the promising diagnostic tool to be used for molecular discrimination of gastric dysplasia. However, as gastric dysplasia belongs to part of a widely accepted multistep, continuum progression cascade from normal gastric tissue to adenocarcinoma (Correa, 1988), it implies vague molecular distinction of gastric dysplasia that may render characterisation and discrimination tougher for Raman spectral analysis. As shown in Figure 3, the Raman spectral pattern between normal and dysplastic gastric tissues could be very similar, it is highly desirable to develop robust diagnostic approaches to extract all possible diagnostic information contained in tissue Raman spectra for well correlation with tissue changes associated with neoplastic transformation. Consequently, both empirical and statistical techniques were explored in this study to attain the likelihood of good clinical discriminators of Raman spectra for separation between normal and dysplastic gastric tissues.

The results of this study confirm that there are specific spectral differences in Raman spectra between dysplasia and normal tissue, demonstrating the utility of NIR Raman spectroscopy in gastric precancer detection. For instance, the relative peak intensities at $1450 \mathrm{~cm}^{-1}\left(\mathrm{CH}_{2}\right.$ proteins/lipids) and $1305 \mathrm{~cm}^{-1}$ (bending mode of $\mathrm{CH}_{3} \mathrm{CH}_{2}$ twisting of protein) (Mahadevan-Jansen and RichardsKortum, 1996; Stone et al, 2000; Huang et al, 2003) were found to be higher for dysplasia tissues, indicating the elevated concentration of biomolecules (e.g., histones) due to hyperchromatism in tissue with dysplastic transformation (Lauwers and Riddell, 1999). In addition, there is also a relative increase of amide III band $\left(1265 \mathrm{~cm}^{-1}\right)$ and amide I band $\left(1655 \mathrm{~cm}^{-1}\right)$ in intensity, suggesting that dysplasia tissue may be associated with an increase in the relative amount of proteins in the $\alpha$-helix conformation. This could be another evidence that there is an increase concentration of histones, the main protein component that makes up the chromatin for dysplasia tissue (Thomas and Prescott, 1977; Huang et al, 2005). A shoulder band at $1660 \mathrm{~cm}^{-1}$ (amide I, $\beta$-pleated sheet, and/or random coil conformation) was also revealed in the difference spectra (Figure 4B), suggesting that dysplastic transformation may also be associated with an increase in the relative amount of protein in the $\beta$-pleated sheet (Huang et al, 2003; Stone et al, 2004). The appearance of these proteins in the $\beta$-pleated sheet conformation may signify more chemical interaction between the proteins and the microenvironment occurring in the cells, which could be related to increase of mitotic activity, one of the cellular alteration characteristics of gastric dysplasia (Correa, 1988). On top of all these, the Raman band at $1335 \mathrm{~cm}^{-1}$ due to the mixture of biochemicals (nucleic acids and proteins due to extracellular matrix) (Stone et al, 2000; Huang et al, 2003) showed slightly higher percentage signals for dysplasia tissue, indicating that the percentage of nucleic acid and protein contents relative to the total Raman-active components is also increased in dysplasia tissue. Raman peak intensity at $875 \mathrm{~cm}^{-1}$ (hydroxyproline of collagen) was found to be much reduced in dysplastic tissue, and this was probably due to the cytoplasmic mucin depletion and the elevated concentration of metalloproteinase, which cleaved collagen in the stroma layer in gastric dysplasia (Correa, 1988; Georgakoudi et al, 2002). On the other hand, the thickening of the epithelium associated with dysplastic progression may attenuate the excitation laser power and also obscure the collagen Raman emission from the deep collagen basal membrane (Badizadegan et al, 2004), thus resulting in a much decrease of Raman signals $\left(875 \mathrm{~cm}^{-1}\right)$ from dysplasia tissue. In addition, the Raman peaks at 1100 and $1745 \mathrm{~cm}^{-1}$ due to phospholipids, and Raman bands for phenylalanine and tryptophan at 1004 and $1210 \mathrm{~cm}^{-1}$, respectively, also showed lower percentage signals for dysplasia tissue compared with the normal tissue, suggesting a decrease in the percentage of phospholipids, phenylalanine, and tryptophan relative to the total Raman-active constituents in the dysplasia (Stone et al, 2000; Huang et al, 2003). The decrease of Raman peak at around $1745 \mathrm{~cm}^{-1}$ associated with dysplastic tissue 
has also been reported in epithelial tissue with malignancies (Ling et al, 2002; Huang et al, 2003, 2005). Therefore, the distinctive differences in Raman spectra between normal and dysplasia tissue further reinforce that Raman spectroscopy can be used to reveal molecular and cellular changes associated with dysplastic transformation.

To develop simple but effective algorithms for identifying abnormal tissue from normal tissue, the nonparametric empirical approach utilising peak intensity or peak intensity ratio measurements of Raman spectra has been widely applied in a number of organ sites to evaluate variations in the tissue spectra associated with malignant changes (Mahadevan-Jansen and Richards-Kortum, 1996; Utzinger et al, 2001; Huang et al, 2003). For example, the ratio of intensities at $1655 \mathrm{~cm}^{-1}(\mathrm{C}=\mathrm{O}$ stretching of collagen and elastin) to $1455 \mathrm{~cm}^{-1}\left(\mathrm{CH}_{2}\right.$ scissoring of proteins and lipids) has been used to spectrally separate tumours from normal tissues in the brain, breast, colon cervix, and the lung (Utzinger et al, 2001; Huang et al, 2003), as both bands are sensitive to histological abnormality (Mahadevan-Jansen and Richards-Kortum, 1996). For differentiation of normal and precancerous tissues, other different intensity bands and ratios such as $\mathrm{I}_{1656}, \mathrm{I}_{1656} / \mathrm{I}_{1325}, \mathrm{I}_{1330} / \mathrm{I}_{1454} v s \mathrm{I}_{1454} / \mathrm{I}_{1656}$, and $\mathrm{I}_{1336} / \mathrm{I}_{1250}$ had also been reported to be of effective diagnostic algorithms for tissue diagnosis and characterisation (Mahadevan-Jansen and Richards-Kortum, 1996; Huang et al, 2003). In this work, selection of different prominent Raman peaks (e.g., peak intensity, bandwidth, and Raman shift) has also been comprehensively explored for gastric tissue classification. On the basis of the difference spectrum between normal and dysplasia tissue (Figure 4B), we found that the nonparametric intensity ratio of Raman peak intensity at $875 \mathrm{~cm}^{-1}$ for hydroxyproline to the peak at $1450 \mathrm{~cm}^{-1}$ for $\mathrm{CH}_{2}$ mode of proteins/lipids was one of the best diagnostic algorithms that yielded a diagnostic sensitivity of $85.7 \%$ and a specificity of $80.0 \%$ for separating dysplasia from normal tissue. The significant difference of the intensity ratio $\left(\mathrm{I}_{875} / \mathrm{I}_{1450}\right)$ between normal and dysplasia tissue may reflect the relative changes in the concentration of potential biological markers from cell surface antigens, cytoplasmic proteins and mucin, collagen in the extracellular matrix, enzymes, and hormones in dysplasia (Correa, 1988; Mahadevan-Jansen and Richards-Kortum, 1996). Further investigation also shows that other intensity ratios including the Raman peak intensity band at $1335 \mathrm{~cm}^{-1}$ (nucleic acids/proteins) with respect to the Raman peak intensities at $1100 \mathrm{~cm}^{-1}$ (phospholipids) and $1745 \mathrm{~cm}^{-1}$ (phospholipids) are also statistically significantly different $(P<0.0001)$ between normal and dysplasia tissue (data not shown). These ratio values are in agreement with histopathologic studies of grading malignancy by the nucleic acid-to-cytoplasm ratio (Lauwers and Riddell, 1999; Huang et al, 2003; Mourant et al, 2005). Hence, the above intensity ratios may also potentially be used as diagnostic algorithms for detecting precancer in the stomach.

The simplistic empirical analysis above only employs a limited number of Raman peaks for tissue diagnosis; most of the information contained in the Raman spectra has not been used for spectral analysis. Since biological tissue is complex, it is likely that there are many biochemical species influencing diseases concurrently. Therefore, a multivariate statistical analysis (e.g., PCA and LDA) (Lachenbruch and Mickey, 1968; Deinum et al, 1999) that utilises the entire spectrum to determine the most diagnostically significant spectral features may improve the diagnostic efficiency of Raman technique for tissue analysis and classification. As such, PCA-LDA together with cross-validation technique was applied in this work to the NIR Raman spectra acquired for dysplasia tissue identification. The unpaired, two-sided Student's $t$-test identified that only a few PCs (PC1, PC2, PC4, and PC5) contained the most diagnostically significant information $(P<0.05)$ for tissue classification. We note that one of the most statistically significant PCs (e.g., PC5) only describes small amount $(2.6 \%)$ of the total variance. This indicates that some PCs with small variances can still contain the useful diagnostic information for revealing molecular changes with dysplastic transformation. However, since the noise present in weak tissue Raman signals may affect the determination of significant PCs with smaller variances for tissue diagnosis (Sasic, 2001), caution should be taken when acquiring the weak tissue Raman signals. Hence, the rapid fibre-optic Raman system with a high S/N ratio (3.3- to 16-fold improvement) (Huang et al, 2001) was employed to obtain highquality Raman tissue spectra, and an appropriate data preprocessing was also introduced for further reducing the noise interference in PC analysis. The consistency in identifying similar, significant PC scores from run to run during the leave-one-spectrum-out, crossvalidation testing suggested that the diagnostic algorithms developed were robust for Raman spectral analysis in this study. To develop effective diagnostic algorithms for tissue classification, all the four diagnostically significant PCs were utilised in the LDA model. The diagnostic sensitivity and specificity of 95.2 and $90.9 \%$, respectively, for identifying dysplasia from normal gastric tissue can be achieved using the PCA-LDA model, which had almost a $10 \%$ improvement in diagnostic accuracy compared with the empirical method. Receiver operating characteristic analysis (Figure 8) further confirms that PCA-LDA-based diagnostic algorithms employing the entire spectral features of Raman spectra are more robust and powerful in distinguishing dysplasia from normal tissue.

It should be noted that PCA is primarily for data reduction rather than for identification of biochemical or biomolecular components of tissue. It is usually difficult to interpret the physical meanings of the component spectra. However, with more powerful diagnostic algorithms (e.g., genetic algorithms) (Mountford et al, 2001), distinctive spectral regions that are optimal for tissue differentiation may be identified and related to particular biochemical and biomolecular changes (e.g., proteins, lipids, nucleic acids, and carbohydrates) associated with neoplastic transformation. These techniques need a much larger Raman data set for robust diagnostic algorithms development. On the other hand, to further understand the relationships between the dysplasia-related morphologic/biochemical changes and the Raman spectra from tissue that is crucial in establishing confidence in clinicians on the application of rapid fibre-optic Raman spectroscopy for precancer detection, confocal Raman microspectroscopy should be explored on the tissue in vivo or in vitro, by measuring the complete Raman spectra of specific tissue microstructures, or alternatively by mapping the distribution of some specific Raman peaks or principal components within a tissue, or even mapping the biochemical distribution at different tissue depth for association with tissue histopathology (Caspers et al, 2003; Shetty et al, 2006). The work in these areas warrants further investigation.

In conclusion, this work shows that significant differences exist in Raman spectra between normal and dysplastic gastric tissue, demonstrating that NIR Raman spectroscopy have the potential to provide diagnostic information necessary for distinguishing precancer from normal tissue. Furthermore, with the development of micron-scale Raman probes for the collection of tissue Raman signals in a few seconds via endoscopy (Shim et al, 2000; Hattori et al, 2007), NIR Raman spectroscopy could be a potentially clinically useful tool for the rapid and noninvasive early diagnosis of gastric precancer in vivo at the molecular level.

\section{ACKNOWLEDGEMENTS}

This research was supported by the Biomedical Research Council, the National Medical Research Council, the Academic Research Fund from Ministry of Education, and the Faculty Research Fund from National University of Singapore. 


\section{REFERENCES}

Axon A (2006) Symptoms and diagnosis of gastric cancer at early curable stage. Best Pract Res Clin Gastroenterol 20(4): 697-708

Badizadegan K, Backman V, Boone CW, Crum CP, Dasari RR, Georgakoudi I, Keefe K, Munger K, Shapshay SM, Sheetse EE, Feld MS (2004) Spectroscopic diagnosis and imaging of invisible pre-cancer. Faraday Discuss 126: $265-279$

Bakker Schut TC, Witjes MJ, Sterenborg HJ, Speelman OC, Roodenburg JL, Marple ET, Bruining HA, Puppels GJ (2000) In vivo detection of dysplastic tissue by Raman spectroscopy. Anal Chem 72: 6010-6018

Bashkatov AN, Genina EA, Kochubey VI, Gavrilova AA, Kapralov SV, Grishaev VA, Tuchin VV (2007) Optical properties of human stomach mucosa in the spectral range from 400 to $2000 \mathrm{~nm}$ : prognosis for gastroenterology. Med Laser Appl 22(2): 95-104

Caspers PJ, Lucassen GW, Puppels GJ (2003) Combined in vivo confocal Raman spectroscopy and confocal microscopy of human skin. Biophys $J$ 85(1): $572-580$

Clark CJ, Thirlby RC, Picozzi Jr V, Schembre DB, Cummings FP, Lin E (2006) Current problems in surgery: gastric cancer. Curr Probl Surg 43: $566-570$

Correa P (1988) A human model of gastric carcinogenesis. Cancer Res 48: $3554-3560$

Deinum G, Rodriquez D, Romer TJ, Fitzmaurice M, Kramer JR, Feld MS (1999) Histological classification of Raman spectra of human coronary artery atherosclerosis using principal component analysis. Appl Spectrosc 53: $938-942$

Devore JL (1992) Probability and Statistics for Engineering and the Science. Pacific Grove: Brooks/Cole

Dillion RW, Goldstein M (1984) Multivariate Analysis: Methods and Applications. New York: John Wiley and Sons

Dollish FR, Fateley WG, Bentley FF (1974) Characteristics Raman Frequencies of Organic Compounds. New York: Wiley

Frank CJ, McCreery RL, Redd DC (1995) Raman spectroscopy of normal and diseased human breast tissues. Anal Chem 67: 777-783

Georgakoudi I, Jacobson BC, Müller MG, Sheets EE, Badizadegan K, CarrLocke DL, Crum CP, Boone CW, Dasari RR, Van Dam J, Feld MS (2002) $\mathrm{NAD}(\mathrm{P}) \mathrm{H}$ and collagen as an in vivo quantitative fluorescence biomarkers of epithelial precancerous changes. Cancer Res 62: 682-687

Gniadecka M, Wulf HC, Nielsen OF, Christensen DH, Hercogova J (1997) Distinctive molecular abnormalities in benign and malignant skin lesions: studies by Raman spectroscopy. Photochem Photobiol 66(4): 418-423

Hattori Y, Komachi Y, Asakura T, Shimosegawa T, Kanai G, Tashiro H, Sata $\mathrm{H}$ (2007) In vivo Raman study of the living Rat esophagus and stomach using a micro-Raman probe under an endoscopy. Appl Spectrosc 61(6): $579-584$

Huang Z, Lui H, Chen XK, Alajlan A, McLean DI, Zeng H (2004) Raman spectroscopy of in vivo cutaneous melanin. J Biomed Opt 9(6): $1198-1205$

Huang Z, Lui H, McLean DI, Korbelik M, Zeng H (2005) Raman spectroscopy in combination with near-infrared autofluorescence background enhances the in vivo assessment of malignant tissues. Photochem Photobiol 81(5): $1219-1226$

Huang Z, McWilliams A, Lui H, McLean DI, Lam S, Zeng H (2003) Nearinfrared Raman spectroscopy for optical diagnosis of lung cancer. Int J Cancer 107: $1047-1052$

Huang Z, Zeng H, Hamzavi I, McLean DI, Lui H (2001) Rapid near-infrared Raman spectroscopy system for real-time in vivo skin measurements. Opt Lett 26: $1782-1784$

Kumar KK, Anand A, Chowdary MVP, Thakur K, Kurien J, Krishna CM, Mathew S (2007) Discrimination of normal and malignant stomach mucosal tissues by Raman spectroscopy: a pilot study. Vib Spectrosc 44: $382-387$

Lachenbruch P, Mickey RM (1968) Estimation of error rates in discriminant analysis. Technometrics 10: 1-11
Lau DP, Huang Z, Lui H, Anderson DW, Berean K, Morrison MD, Shen L, Zeng $\mathrm{H}$ (2005) Raman spectroscopy for optical diagnosis in the larynx - preliminary findings. Lasers Surg Med 37(3): 192-200

Lauwers GY, Riddell RH (1999) Gastric epithelial dysplasia. Gut 45: $784-790$

Ling XF, Xu YZ, Weng SF, Li WH, Xu F, Hammaker RM, Fateley WG, Wang F, Zhou XS, Soloway RD, Ferraro JR, Wu JG (2002) Investigation of normal and malignant tissue samples from the human stomach using Fourier transform Raman spectroscopy. Appl Spectrosc 56(5): 570 - 573

Mahadevan-Jansen A, Mitchell MF, Ramanujam N, Malpica A, Thomsen S, Utzinger U, Richards-Kortum R (1998a) Near-infrared Raman spectroscopy for in vitro detection of cervical precancers. Photochem Photobiol 68: $123-132$

Mahadevan-Jansen A, Mitchell MF, Ramanujam N, Utzinger U, RichardsKortum R (1998b) Development of a fiber optic probe to measure NIR Raman spectra of cervical tissue in vivo. Photochem Photobiol 68: $427-431$

Mahadevan-Jansen A, Richards-Kortum R (1996) Raman spectroscopy for the detection of cancers and precancers. J Biomed Opt 1: $31-70$

Mizuno A, Kitajima H, Kawauchi K, Muraishi S, Ozaki Y (1994) Nearinfrared Fourier transform Raman spectroscopic study of human brain tissues and tumors. J Raman Spectrosc 25: 25-29

Molckovsky A, Song LM, Shim MG, Marcon NE, Wilson BC (2003) Diagnostic potential of near-infrared Raman spectroscopy in the colon: differentiating adenomatous from hyperplastic polyps. Gastrointest Endosc 57: 396-402

Mountford CE, Somorjai RL, Malycha P, Gluch L, Lean C, Russell P, Barraclough B, Gillett D, Himmelreich U, Dolenko B, Nikulin AE, Smith IC (2001) Diagnosis and prognosis of breast cancer by magnetic resonance spectroscopy of fine-needle aspirates analysed using a statistical classification strategy. Br J Surg 88: 1234-1240

Mourant JR, Short KW, Carpenter S, Kunapareddy N, Coburn L, Powers TM, Freyer JP (2005) Biochemical differences in tumorigenic and nontumorigenic cells measured by Raman and infrared spectroscopy. J Biomed Opt 10(3): 031106

Sabet EA, Okai T, Minamoto T, Mai M, Sawabu N (2003) Visualizing the gastric wall with a $30-\mathrm{MHz}$ ultrasonic miniprobe: ex vivo imaging of normal gastric sites and sites of early gastric cancer. Abdom Imaging 28(2): $252-256$

Sasic S (2001) Eigenvalues and principal component loadings or heavily overlapped vibrational spectra. Spectrochim Acta A 57: $323-336$

Shetty G, Kendall C, Shepherd N, Stone N, Barr H (2006) Raman spectroscopy: elucidation of biochemical changes in carcinogenesis of oesophagus. Br J Cancer 94: 1460-1464

Shim MG, Song LM, Marcon NE, Wilson BC (2000) In vivo near-infrared Raman spectroscopy: demonstration of feasibility during clinical gastrointestinal endoscopy. Photochem Photobiol 72: $146-150$

Stone N, Kendall C, Sheperd N, Crow P, Barr H (2002) Near-infrared Raman spectroscopy for the classification of epithelial pre-cancers and cancers. J Raman Spectrosc 33: 564-573

Stone N, Kendall C, Smith J, Crow P, Barr H (2004) Raman spectroscopy for identification of epithelial cancers. Faraday Discuss 126: $141-157$

Stone N, Stavroulaki P, Kendall C, Birchall M, Barr H (2000) Raman spectroscopy for early detection of laryngeal malignancy: preliminary results. Laryngoscope 110: 1756-1763

Teh M, Tan KB, Seet BL, Yeoh KG (2002) Study of p53 immunostaining in the gastric epithelium of CagA-positive and CagA-negative helicobacter pylori gastritis. Cancer 95(3): 499-505

Thomas Jr GJ, Prescott B (1977) Secondary structure of histones and DNA in chromatin. Science 197(4301): $385-388$

Utzinger U, Heintzelman DL, Mahadevan-Jansen A, Malpica A, Follen M, Richards-Kortum R (2001) Near-infrared Raman spectroscopy for in vivo detection of cervical precancers. Appl Spectrosc 55: 955-959 